\title{
Article
}

\section{Qu'est-ce que l'emploi durable?}

\author{
Sébastien Knockaert ${ }^{\mathrm{a}}$, Muriel Maillefert ${ }^{\mathrm{b}}$ \\ a Doctorant en économie, FORUM, Université de Paris 10, 200 av. de la République, 92001 Nanterre cedex, France \\ b Économiste, Université Lille 3, BP 149, 59653 Villeneuve d'Ascq cedex, France
}

NSS revient à nouveau sur la question du développement durable. Encore une fois pourrait-on dire, bien que ce soit sous l'angle inédit de l'emploi. Parce que les auteurs, confrontés à la vacuité du lien entre durabilité et emploi, décident de voir ce qui se joue concrètement dans les emplois locaux se réclamant de l'environnement, ils se posent en filigrane une question essentielle : le développement durable est-il possible dans un contexte mental et productif inchangé ? À l'heure où certains explorent l'idée d'une décroissance, cet article offre, en confrontant les dynamiques de l'emploi et les critères de l'utopie durable, une réflexion très opportune qui prolonge le débat ouvert depuis plusieurs années par la revue.

La Rédaction

\section{Mots-clés :}

développement durable ; emploi ; développement territorial ; politiques d'emploi

\section{Keywords:}

Sustainable development; employment; local policies; employment policies
Résumé - Les préoccupations actuelles en matière d'emploi (montée du chômage, notamment des non-qualifiés, problèmes d'exclusion) laissent penser que la problématique du développement durable, à la croisée de l'économique, du social et de l'environnemental est apte à définir l'emploi durable. Une étude des différentes définitions du développement durable montre que l'emploi y est peu ou pas pris en charge. L'approche économique s'inscrit en effet dans une problématique particulière, dans laquelle le facteur principal à prendre en compte est le capital. De même, les approches plus localisées ne donnent aucun contenu à l'emploi durable, même si celui-ci est considéré comme un facteur important. Il convient donc de repartir d'expériences concrètes comme l'analyse des emplois-environnement. On se heurte alors à la très grande hétérogénéité de ces emplois et à l'ambiguïté de leur inscription dans plusieurs espaces : ceux du secteur marchand, où la référence est l'emploi normal, et ceux de la sphère non marchande, dont la préoccupation est l'insertion, et pour laquelle la durabilité se confond souvent avec la pérennisation des emplois. Au total, trois caractéristiques apparaissent importantes pour caractériser l'emploi durable : la qualité des emplois, l'investissement territorial et la gestion partenariale.

\footnotetext{
Abstract - What is sustainable employment? The example of environmental jobs. The paper aims at defining sustainable jobs. Sustainable jobs are referred to sustainable growth where social, environmental and economic conditions are satisfied. First, different definitions of growth are examined. We show that the economic definitions don't get the question of employment right: labour is missing because full employment is supposed in the long run. Then, local experiences of sustainable employment are examined. Two kinds of jobs are found. On the one hand, jobs that belong to the market sector and refer to normal jobs (full time jobs kept by insiders). These jobs are not connected with the question of sustainability. On the other hand are found jobs which refer to the public employment policy, which aim at helping unemployed people to find a new occupation. These jobs refer to transitional markets or the non profit sector. These jobs are numerous in the environmental sector. But most of them are temporary and belong to the so called secondary sector. People who have these jobs want them to become permanent jobs, but don't question their quality or characteristics as sustainability stands. Then sustainability is confused with stability, and the quality of jobs is not examined. In fact, since these policies need a local grounding, there is confusion between the goals of environmental policies and employment policies, which obscure what is really at stake in the development of sustainable jobs: a definition of new economic constraints or a tool for public policies (either centralised or decentralised). Finally, three characteristics are suggested: quality of jobs, local commitment and partnership.
} 
Selon les termes bien connus du rapport Brundtland (WECD, 1987), le développement durable (DD par la suite) vise à assurer conjointement le bien-être (inter- et intra-générationnel) des individus, une répartition équitable du surplus ainsi que la conservation des ressources environnementales. Ces trois dimensions, économique, sociologique et environnementale, paraissent particulièrement bien adaptées à l'analyse de l'emploi, qui semble à l'évidence pouvoir relever de chacune d'entre elles. Pourtant, définir l'emploi durable est difficile ${ }^{1}$. Une démarche naturelle consiste à partir des définitions économiques $\mathrm{du}$ DD pour tenter d'en déduire une caractérisation de l'emploi durable. Cette démarche reste cependant décevante parce que les définitions du DD, basées principalement sur les contraintes concernant l'utilisation des ressources (en tant que prélèvement sur un stock disponible et en tant que rejet hors du système économique), ne sont pas centrées sur l'emploi. De plus, les approches économiques modélisées du développement durable sont trop abstraites et ne peuvent, par construction, fournir autre chose qu'une méthodologie générale.

Or, le concept d'emploi durable dont il s'agit ici fait référence à un contexte précis, qui est celui de la lutte contre le chômage dans les pays industrialisés ${ }^{2}$, et plus particulièrement dans des régions anciennement industrialisées (comme la région Nord-Pas-de-Calais). Ces régions cumulent l'ensemble des conséquences négatives du déclin industriel : des niveaux de chômage élevés et persistants ainsi qu'une dégradation importante de l'environnement (Chautard, 1997, 2000) ${ }^{3}$. Un des objectifs de la politique économique locale est alors d'assurer conjointement le développement de l'emploi et la durabilité de la croissance, durabilité signifiant à la fois retour d'une croissance économique positive dans la durée, et effort pour que cette croissance soit respectueuse de l'environnement (Conseil régional Nord-Pas-de-Calais, 1996, 2000).

Reste à caractériser l'emploi associé à cette configuration de la croissance, que nous appelons emploi durable.

\footnotetext{
${ }^{1}$ Ce travail est l'aboutissement d'une interrogation sur l'opportunité de croiser la problématique du développement durable et de l'emploi. Certaines des questions ont été discutées dans le cadre d'un séminaire du DESS «Gestion et protection de l'environnement » à Lille 3. Une enquête a été menée dans la région Nord-Pas-de-Calais sur les activités d'insertion par l'environnement, et elle constitue le point de départ d'un travail plus conséquent sur la thématique développement durable et territoire. Une première version du texte a été proposée aux journées NSS «De l'écologie urbaine à la ville durable, quels besoins de recherche pour quelles pratiques interdisciplinaires?» (Engref, Paris, 6-7 décembre 2000).

2 Nous faisons référence aux approches récentes du DD, qui concernent les pays industrialisés, et non à celles, plus anciennes, concernant les pays en développement.

${ }^{3}$ Le terme de "région sacrifiée » est parfois utilisé pour caractériser ces régions (Zuindeau, 1997b).
}

Peut-on trouver des propriétés générales permettant de le définir? Plusieurs méthodes sont successivement envisagées.

La première consiste à rechercher des propriétés générales pouvant caractériser cet emploi dans les travaux formels sur le développement durable. Compte tenu de ses résultats décevants, elle est complétée par une analyse plus appliquée des emplois associés au secteur environnemental. Les observations empiriques font apparaître une grande hétérogénéité des formes d'emploi de ce secteur et l'ambiguïé des caractéristiques de ces emplois en matière de durabilité. Cette difficulté conduit, de fait, à proposer des critères transversaux, permettant de parler de configurations de l'emploi congruentes avec les définitions du $\mathrm{DD}^{4}$.

\section{La définition de l'emploi durable dans les approches économiques globales}

Le DD étant un concept macro-économique, on est autorisé à rechercher comment est intégré l'emploi dans les définitions économiques du DD. Cette méthode donne des résultats assez décevants, parce que l'emploi est considéré comme une conséquence de la croissance et n'est pas analysé pour lui-même, sauf dans des cas très particuliers. D'autres critères, plus empiriques, doivent ainsi être mobilisés, mais ils ne se montrent, à leur tour, pas totalement satisfaisants.

\section{L'approche modélisée du DD : l'emploi comme conséquence de la croissance}

Pezzey a recensé en 1989 l'essentiel des travaux modélisés sur le développement durable ${ }^{5}$. Une analyse textuelle des définitions présentées en annexe de son modèle montre que le développement durable est défini essentiellement en relation avec la question des ressources naturelles et, de façon moins nette, de la croissance. La multidimensionnalité de la durabilité est donc limitée à ces critères. Les « ressources naturelles » sont principalement caractérisées par leurs propriétés physiques, à savoir leur caractère épuisable ou renouvelable. Le capital est la variable assurant le lien entre les ressources et

\footnotetext{
${ }^{4}$ Le secteur non marchand est essentiellement pensé en référence aux politiques publiques de l'emploi. On sait que ces politiques peuvent avoir des points communs avec les activités du tiers secteur, mais celui-ci n'est pas analysé en tant que tel.

5 À noter que Pezzey (1989) se limite explicitement aux définitions néoclassiques du développement durable, à la différence d'autres synthèses comme par exemple celle proposée par le Cirad. Cependant, parmi les 50 auteurs recensés par cette dernière, aucun ne cite explicitement l'emploi.
} 
la croissance : les ressources font partie du stock de capital à conserver. On retrouve ainsi, exprimé de manière implicite, le critère de la soutenabilité faible ${ }^{6}$.

Une des raisons essentielles de l'absence de référence à l'emploi tient à l'horizon temporel utilisé : comme pour les théories de la croissance, l'horizon temporel est le long terme (Pearce et Atkinson, 1998) et l'économie est considérée de fait comme étant au plein emploi. Cette dernière hypothèse est importante pour notre propos, car elle signifie clairement, et justifie en elle-même, l'absence de discussion sur l'emploi (Pezzey, 1989).

Toutefois, dans un contexte de chômage élevé et persistant, et de sous-emploi, une telle position ne paraît pas satisfaisante. En fait, les travaux spécifiques qui modélisent les relations entre environnement et emploi ne la retiennent pas. Cependant, les modèles s'interrogent essentiellement sur l'aspect quantitatif de l'emploi (le volume d'emploi) mais non sur son aspect qualitatif (les caractéristiques de l'emploi). L'emploi est ainsi analysé comme une conséquence de l'activité économique et l'objectif de la politique économique est d'augmenter le volume d'emploi, éventuellement dans le secteur environnemental (OCDE, 1997; Roch, 1996, pour un recensement général des modèles d'emploi; Grimal et Kephaliacos, 2000, pour le développement original d'un modèle d'écologie industrielle).

Une famille particulière de modèles traite du lien entre politique fiscale et emploi, à travers la thématique du «double dividende» (Bureau et Hourcade, 1998). L'idée principale est qu'un recyclage approprié des taxes sur les émissions de $\mathrm{CO}_{2}$ peut sous certaines conditions augmenter la demande de travail non qualifié et promouvoir, au-delà des effets environnementaux eux-mêmes, $l^{\prime}$ emploi ${ }^{7}$. Cependant, les effets nets sur l'emploi sont incertains du fait notamment de la complexité des effets induits (hausse éventuelle des salaires, des prix, substitutions intersectorielles) (Lepeltier, 1999) ${ }^{8}$. Au total,

\footnotetext{
${ }^{6}$ Le critère de la soutenabilité faible est construit à partir de l'hypothèse d'une substituabilité possible entre les différentes formes de capital (le capital naturel étant une composante du stock total de capital, qui inclut également d'autres formes de capital comme le capital manufacturé et le capital humain). Selon ce critère, le stock agrégé de capital doit être maintenu constant, sans condition spécifique sur sa composition (Faucheux et Noël, 1995 ; Bontems et Rotillon, 1998).

7 Dans l'acception généralement admise, le premier dividende concerne l'internalisation des effets négatifs sur l'environnement par les entreprises. L'écotaxe incite ainsi les entreprises à modifier leur technologie vers une technologie plus respectueuse de l'environnement. Le second dividende concerne le recyclage de la taxe en faveur de l'emploi non qualifié, par exemple en diminuant les charges sociales sur les emplois en question (voir CAE $n^{\circ} 8,1998$ ).

8 Les effets en équilibre partiel sont en général ceux qui sont étudiés en premier : par exemple, toutes choses égales par ailleurs, baisser les cotisations sociales fait monter la demande
}

le caractère « sans regret ${ }^{9}$ » de telles mesures semble plus convaincant que leur incidence sur l'emploi.

Au-delà de ces quelques exceptions, les modèles traitent rarement de la problématique de l'emploi en tant que telle. On peut en trouver la raison dans la manière dont ils sont construits : les modèles ne s'interrogent pas sur la nature de la croissance. Comme l'avait déjà mentionné Pezzey (1989), la durabilité a le statut de contrainte introduite dans un contexte économique global inchangé. Cette contrainte peut entraîner des effets positifs, par exemple des effets redistributifs en faveur des ménages moins aisés ou la création d'emplois, ou négatifs, en générant des délocalisations d'entreprises ou en diminuant la productivité. Quelle que soit leur nature, ces effets se réalisent dans un contexte productif inchangé.

L'examen des modèles économiques montre qu'il n'existe pas, à cette échelle, de définition qualitative de l'emploi permettant d'avancer une définition de l'emploi durable. L'emploi est considéré comme une variable d'état, qui dérive directement de la croissance (ou du développement). La question pertinente est celle du contenu en emplois de la croissance (via éventuellement une discussion sur les technologies de production) dans lequel $x$ points de croissance vont donner $y$ points d'emploi. Une politique économique favorable à l'emploi s'efforcera alors de rendre la croissance "plus riche en emplois».

Au total, les modèles économiques apparaissent mal armés pour aborder la question de l'emploi en général et celle de l'emploi durable en particulier. Qu'en est-il des approches plus appliquées, qui examinent les emplois relevant du secteur environnemental?

\section{Les approches appliquées : une première caractérisation des emplois-environnement}

À la différence des modèles formels, qui s'intéressent aux fonctions de production dans leur généralité, les approches appliquées croisent les thématiques de l'emploi et de l'environnement sous forme d'une étude des «emplois-environnement», tels que les définissent les statistiques officielles. Celles-ci les abordent par un découpage en grand secteur d'activité (eau, déchets, etc.) (Ifen, 1999a, 1999b). Les informations qualitatives y sont cependant assez frustes, même s'il existe des données

de travail. Mais si les taxes se traduisent par exemple par une hausse des prix ou des salaires, les effets en équilibre général (combinant plusieurs marchés, comme le marché des biens et du travail par exemple) deviennent très difficiles à évaluer.

9 L'aspect «sans regret» de la politique compare son efficacité en regard d'autres instruments, indépendamment de son effet recherché (ici environnemental). Il s'agit alors d'évaluer, dans l'absolu, l'effet comparé d'instruments de politique économique (par exemple, dans notre cas, comparer l'effet sur l'activité d'une écotaxe par rapport à un autre prélèvement fiscal). 
Tableau. Activités, acteurs et formes d'emploi de l'environnement.

\begin{tabular}{|c|c|c|c|c|c|}
\hline $\begin{array}{l}\text { Type } \\
\text { d'activité }\end{array}$ & $\begin{array}{l}\text { Activités lourdes à } \\
\text { fort contenu en } \\
\text { capital technique, } \\
\text { bien spécifiées } \\
\text { juridiquement et } \\
\text { protégées de la } \\
\text { concurrence } \\
\text { internationale }\end{array}$ & $\begin{array}{l}\text { Activités plus } \\
\text { diffuses de } \\
\text { dépollution, } \\
\text { requérant } \\
\text { éventuellement } \\
\text { des technologies } \\
\text { de pointe, } \\
\text { possibilité } \\
\text { d'ouverture à la } \\
\text { concurrence } \\
\text { internationale }\end{array}$ & $\begin{array}{l}\text { Activités de } \\
\text { service } \\
\text { dépendant } \\
\text { traditionnelle- } \\
\text { ment de la } \\
\text { sphère } \\
\text { publique }\end{array}$ & $\begin{array}{l}\text { Activités } \\
\text { répondant à une } \\
\text { demande sociale } \\
\text { plus ou moins } \\
\text { affirmée, moins } \\
\text { bien cernées sur } \\
\text { le plan juridique, } \\
\text { avec des aspects } \\
\text { incomplets de } \\
\text { solvabilisation }\end{array}$ & $\begin{array}{l}\text { Activités } \\
\text { environnementales } \\
\text { exercées } \\
\text { dans des } \\
\text { secteurs divers } \\
\text { pour des raisons } \\
\text { économiques } \\
\text { (réduction des } \\
\text { coûts, gestion de } \\
\text { l'image) }\end{array}$ \\
\hline $\begin{array}{l}\text { Domaine } \\
\text { d'activité }\end{array}$ & $\begin{array}{l}\text { Mobilisation de la } \\
\text { ressource en eau, } \\
\text { épuration des eaux } \\
\text { résiduaires, } \\
\text { traitement standard } \\
\text { des déchets }\end{array}$ & $\begin{array}{l}\text { Production de } \\
\text { «technologies } \\
\text { propres»: } \\
\text { dispositifs de } \\
\text { dépollution de l'air, } \\
\text { techniques de } \\
\text { protection contre le } \\
\text { bruit, services } \\
\text { privés de } \\
\text { l'environnement }\end{array}$ & $\begin{array}{l}\text { Nettoyage des } \\
\text { rues, entretien } \\
\text { d'espaces verts }\end{array}$ & $\begin{array}{l}\text { Valorisation des } \\
\text { déchets, entretien } \\
\text { des paysages }\end{array}$ & $\begin{array}{l}\text { Activités } \\
\text { polluantes ou } \\
\text { sensibles sur le } \\
\text { plan } \\
\text { environnemental }\end{array}$ \\
\hline $\begin{array}{l}\text { Acteurs } \\
\text { principaux }\end{array}$ & Grandes entreprises & PME/PMI vertes & $\begin{array}{l}\text { Collectivités } \\
\text { locales }\end{array}$ & $\begin{array}{l}\text { Associations et } \\
\text { entreprises } \\
\text { intermédiaires }\end{array}$ & $\begin{array}{l}\text { Grandes } \\
\text { entreprises }\end{array}$ \\
\hline $\begin{array}{l}\text { Type } \\
\text { d'emploi }\end{array}$ & $\begin{array}{l}\text { Emploi normal. Pas } \\
\text { de gestion spécifique } \\
\text { de l'emploi- } \\
\text { environnement }\end{array}$ & $\begin{array}{l}\text { Emploi normal. } \\
\text { Caractère } \\
\text { expérimental de } \\
\text { certaines activités : } \\
\text { recours à des } \\
\text { contrats atypiques }\end{array}$ & $\begin{array}{l}\text { Emploi normal } \\
\text { et recours } \\
\text { important aux } \\
\text { dispositifs aidés } \\
\text { (logique } \\
\text { insertive) }\end{array}$ & $\begin{array}{l}\text { Emploi normal et } \\
\text { recours important } \\
\text { aux dispositifs } \\
\text { aidés (logique } \\
\text { insertive et de } \\
\text { création de } \\
\text { nouvelles } \\
\text { activités) }\end{array}$ & $\begin{array}{l}\text { Emploi normal } \\
\text { mais problème } \\
\text { de taille du } \\
\text { service } \\
\text { environnement. } \\
\text { Recours possible } \\
\text { aux contrats } \\
\text { atypiques (CDD, } \\
\text { stagiaires) pour } \\
\text { ces emplois }\end{array}$ \\
\hline
\end{tabular}

Source : d'après Zuindeau (1996).

(encore embryonnaires) sur les statuts des emplois de l'environnement (contrat à durée indéterminée, CDI, ou contrat à durée déterminée, CDD) (Ministère de l'Aménagement du territoire et de $1^{\prime}$ Environnement, 1997) ${ }^{10}$.

Dans une optique légèrement différente, plus centrée sur l'activité, Zuindeau (1996) cherche à recenser le potentiel en emplois du secteur environnemental. Pour ce

\footnotetext{
${ }^{10}$ Les informations qualitatives sur les emplois sont en effet peu abondantes et difficiles d'accès. Elles concernent le statut de l'emploi (CDI ou CDD) et le métier (décliné en quatre modalités) et sont fournies par l'ANPE (Agence nationale pour l'emploi), l'APEC (Agence pour l'emploi des cadres) en termes d'offres d'emploi, et l'INSEE (DADS, Déclarations annuelles des salaires opérées par les entreprises). L'Ifen dispose d'une base de données sur les emplois-environnement et édite un journal électronique, $\mathrm{La}$ Feuille de l'ORME, consacré à cette thématique.
}

faire, il s'appuie sur une déclinaison des activités de protection de l'environnement (Tableau). Ainsi, aux activités sont associés des domaines et des acteurs (lignes 1 à 3 du tableau). Il est alors aisé d'y greffer une typologie des caractéristiques statutaires des emplois, que nous appelons, suivant la terminologie en vigueur, types d'emploi (ligne 4). On voit apparaître sans surprise les différentes déclinaisons statutaires habituelles.

Les éco-industries (colonnes 2 et 3 ) se situent de fait dans le secteur environnemental, sans qu'y soit développé de problématique spécifique concernant l'emploi : comme ailleurs on y trouve des emplois normaux en CDI ou toute autre forme atypique d'emploi. C'est le cas aussi des entreprises privées mettant en œuvre des actions en faveur de l'environnement (normalisation, labellisation), voire d'accès à l'emploi (actions d'insertion), qui font partie d'une démarche globale, mais qui n'ont pas d'activité 
environnementale à proprement parler (colonne 6). L'action de ces entreprises peut être jaugée à l'aune de critères sociaux et environnementaux, on y reviendra plus tard. L'emploi de référence y est pourtant sans conteste l'emploi normal.

À côté de la sphère privée se développent des activités contrôlées ou impulsées par le secteur public, qui utilisent fortement les contrats aidés, que ce soit pour favoriser la création d'activités nouvelles ou enclencher la pérennisation d'activités existantes (colonnes 4 et 5). Ces activités ont comme particularité d'employer des individus à la fois sous contrat de travail normal et sous contrat aidé. Elles peuvent être liées à un projet environnemental explicite, voire une politique de développement durable qui s'affiche comme telle. C'est par exemple le cas de la politique emploi-environnement développée par le conseil régional du Nord-Pas-de-Calais qui se déploie essentiellement dans le domaine de la reconquête et du maintien des espaces naturels et du cadre de vie. La composante se référant au DD y est explicitement formulée sous forme de contraintes imposées aux projets qui soumissionnent à une aide financière : les projets doivent être présentés par des acteurs locaux (les communes, les structures intercommunales et associations) et sont jugés sur des critères explicites de développement durable (pertinence écologique, pertinence sociale, perspectives de développement vers une activité pérenne, valeur en termes d'exemplarité, volonté partenariale, nonconcurrence avec les entreprises du secteur privé ou avec la fonction publique). Mais ces critères s'accompagnent d'exigences qualitatives assez réduites sur les emplois offerts. Elles se cantonnent d'une part à la nature du contrat de travail (CDI, CDD d'au moins un an ou contrat emploi consolidé), à la formation à donner au personnel bénéficiaire de l'aide et, enfin, au domaine d'activité principal (l'occupation doit être de nature environnementale). À noter qu'à l'échelle de la région, ces projets concernent un volume non négligeable d'emplois, estimé à 1000 (en 2000) par le conseil régional ${ }^{11}$.

Les exemples précédents témoignent ainsi de la difficulté à déterminer des critères exhaustifs pour évaluer la durabilité des emplois. On ne peut, en effet, se contenter du découpage traditionnel entre emploi en CDI et emploi précaire.

Dans le travail cité précédemment, Zuindeau (1996) propose de retenir trois critères d'évaluation pour les emplois qu'il a identifiés comme emplois-environnement, qui concernent à la fois les activités environnementales et les emplois qui y sont associés : la durabilité (appuyée sur la pérennité des activités), la qualité (fondée sur la qualification et la transférabilité des compétences acquises) et

\footnotetext{
11 Dont $60 \%$ en contrat aidé, $40 \%$ sous statut normal, pour un total de 120 projets.
}

enfin, l'efficience économique (évaluée à partir de critères de réduction des coûts sociaux de long terme).

Ces critères, essentiellement rattachés à l'activité, présentent l'avantage de compatibilité avec le cadre du développement durable. Toutefois, leur interprétation en termes d'emploi est malaisée. Car si les conditions 2 et 3 (qualité et efficience) font explicitement référence à des propriétés qualitatives du travail ou de l'emploi, qui semblent en effet pertinentes, la condition 1 (les emplois sont durables parce que reposant sur des activités pérennes) apparaît insatisfaisante. Elle peut en effet se comprendre comme une référence simple au cadre du modèle d'emploi normal, qui est lui-même en déclin et très contesté sur l'aspect qualitatif (Pisani-Ferry, 2000).

C'est pourquoi, même si ces travaux constituent un point de départ appréciable, d'autres critères doivent être explorés.

\section{Pistes pour une caractérisation de l'emploi durable}

Comme le montre la typologie précédente, les emplois-environnement concernent tout le spectre de l'économie, du secteur marchand au secteur non marchand. Ils peuvent cependant, en forçant un peu le trait, être regroupés en deux grandes catégories : les emplois marchands, liés à une problématique de "pérennisation », et les emplois non marchands issus de la politique de l'emploi, liés à une logique d' «insertion ». Dans le premier cas, l'enjeu est de transformer des emplois éventuellement précaires en emplois normaux (le cas limite étant l'accès direct à un emploi stable dans le secteur environnemental). Dans le second cas, les emplois, toujours temporaires, ont vocation à constituer des passerelles pour des individus en situation de chômage, l'horizon étant également, mais de manière moins certaine, l'accès à un emploi normal.

L'accrochage de l'emploi-environnement à des caractéristiques liées à des figures de l'emploi de la période de croissance (référence à l'emploi normal, référence à un secteur d'activité fixe, ici, environnemental) peut paraître nécessaire, mais conduit peut-être à une impasse, dans la mesure où une seule composante est activée à la fois (le secteur, le statut, la pérennité). Or, le DD n'est pas rattaché à une composante fixe, qu'elle soit statutaire ou sectorielle, mais à l'articulation de plusieurs dimensions.

On peut ainsi faire l'hypothèse que ce qu'il importe d'identifier, c'est la manière dont s'articulent les différentes dimensions du DD dans l'emploi. De ce point de vue, il apparaît que les emplois marchands et non marchands ne s'opposent pas, mais présentent des modalités spécifiques d'articulation des différentes dimensions du DD. 


\section{Logiques marchande et non marchande : une combinaison spécifique des trois dimensions du DD}

Le DD suppose l'articulation entre les dimensions de l'économique, de l'environnement et du social. Il n'est pas absurde de supposer que dans le secteur marchand, l'efficacité économique prime sur les deux autres dimensions, alors que le secteur non marchand se singularise par la prévalence de la variable sociale (en assimilant l'insertion à la dimension sociale). C'est ce qui explique les configurations très différentes auxquelles aboutissent les démarches privées marchandes et publiques non marchandes.

Pour bien montrer ces différences, et sans vouloir prétendre à l'exhaustivité, deux exemples, qui semblent typiques, seront développés : la démarche de responsabilité sociale et environnementale (RSE) pour le secteur marchand, certains dispositifs de politique d'emploi pour le secteur non marchand. L'identification de certains aspects saillants permettra d'avancer des critères plus généraux de définition de l'emploi durable.

\section{La dominante environnementale : les démarches de type RSE}

Le Conference Board (Canada) définit la RSE comme «l'ensemble des relations que la firme entretient avec toutes ses parties prenantes : les clients, les employés, la communauté, les actionnaires, les gouvernements, les fournisseurs et les concurrents. Les éléments de la responsabilité sociale incluent l'investissement dans la communauté, les relations avec les employés, la création et le maintien d'emploi, les préoccupations environnementales et la performance financière » (Gendron, 2002, p. 2).

La RSE apparaît comme une démarche volontaire des entreprises pour développer des critères compatibles avec les attentes de parties prenantes (stakeholders), considérées comme différentes, voire parfois opposées, aux attentes des seuls actionnaires (shareholders), dont l'objectif économique se réduit à la maximisation du profit.

Comment justifier une telle modification du comportement des entreprises, manifestement contraire à la vision rationnelle de l'activité économique et au comportement habituel des entreprises? On sait, par exemple, que l'intégration des aspects sociaux par les entreprises, historiquement première, s'est le plus souvent effectuée de manière conflictuelle et sous la contrainte de la législation (Caire, 2003).

L'inflexion vers une prise en compte d'objectifs autres que la recherche du profit, seule responsabilité de l'entreprise selon la théorie orthodoxe, est récente (Friedman, 1970 , p. 11). On peut l'expliquer par des critères extraéconomiques, comme le propose la théorie des parties prenantes. Selon cette théorie, l'entreprise se doit d'être responsable face à toutes les parties concourant à son activité, comme ses actionnaires ou ses salariés. L'environnement apparaît comme une variable supplémentaire rattachée à cette thématique, ce qui suppose, de manière plus discutable, de considérer l'environnement comme une partie prenante (Starik, 1994) ${ }^{12}$.

Une autre manière $\mathrm{d}$ 'aborder la question est de l'intégrer dans un argumentaire économique. Un élargissement des hypothèses sur le comportement des agents et le fonctionnement des marchés le permet. Ainsi, l'introduction d'une hypothèse de concurrence imparfaite rend rationnelle pour les entreprises la recherche d'une internationalisation d'un certain nombre de risques (autrefois analysés comme des disfonctionnements du marché), de manière à en minimiser le coût. Le risque principal auquel les entreprises doivent faire face dans un tel contexte est un risque de réputation. La réalisation d'un tel risque peut occasionner des coûts directs (par exemple en cas de procès suite à une catastrophe environnementale ou, dans le domaine social, en cas de grève des salariés), ou indirects (en cas de défection des clients entraînant une perte de part de marché).

Les entreprises font face à ce risque d'élévation des coûts par une politique de communication appropriée ${ }^{13}$, qui doit appuyer et valoriser l'évolution de leurs pratiques. Ces dernières peuvent elles-mêmes se traduire par des gains nets, autant sur le plan environnemental (gains sur les consommations intermédiaires d'énergie ou d'eau, ou sur les coûts d'élimination des déchets), que social (meilleure productivité des salariés entraînant un gain d'efficacité $\left.{ }^{14}\right)$.

Vue sous cet angle, la problématique de la RSE a donc un sens dans chacun des domaines concernés (social ou environnemental). Elle se justifie par une déclinaison spécifique de la problématique des externalités, soit dans le domaine environnemental, soit dans le domaine social.

Dans les faits, elle se décline en deux grandes familles : la première met l'accent sur l'aspect social (par exemple dans le cas de la démarche de "travail décent » du Bureau international du travail (BIT, 1999) ${ }^{15}$ dont dérive

\footnotetext{
${ }^{12}$ La seule question laissée en suspens, selon cet auteur, porte sur qui le représentera et défendra ses intérêts.

13 Cette thématique rejoint d'ailleurs un ensemble de travaux sur les politiques de communication des entreprises et les externalités (Depoers et Maillefert, 2001).

14 Selon les nouvelles théories du salaire qui relient salaire, effort et productivité. La plus connue d'entre elle est la théorie du salaire d'efficience de Shapiro-Stiglitz.

${ }^{15}$ La démarche de l'OIT (Organisation internationale du travail) rejoint la problématique du PNUD (Programme des Nations Unies pour le développement) qui s'exprime sous forme $\mathrm{d}^{\prime}$ « Objectifs millénaires pour le développement » (OMD), au nombre de cinq : 1) réduction de moitié de l'extrême pauvreté et la faim ; 2) réalisation de l'éducation primaire universelle et de l'égalité des sexes ; 3) réduction des deux tiers de la mortalité
} 
la norme SA 8000 promulguée par le Social Accountabilty International $\left.{ }^{16}\right)$, alors que la seconde tente d'articuler normes sociales et environnementales (c'est le cas de la démarche Global Compact soutenue par l'ONU, ou, plus modestement, de la démarche qui sous-tend l'article 116 de la loi française sur les nouvelles régulations économiques).

Malgré une homogénéité théorique en termes d'externalité, les démarches de type RSE peinent, dans leur mise en œuvre, à articuler de manière cohérente les aspects sociaux et environnementaux, le second aspect apparaissant paradoxalement plus aisé à mettre en œuvre ${ }^{17}$ et plus consensuel, au moins à l'échelle internationale (Cossart et Plihon, 2003).

Nous verrons par la suite, et notamment par comparaison avec le second type de démarche, qui concerne cette fois plus directement l'action publique, ce que ces différentes expériences peuvent avoir d'intéressant en ce qui concerne la définition de l'emploi durable.

\section{La dominante sociale : les démarches de politique publique d'emploi}

La politique publique de l'emploi constitue l'un des terrains privilégiés d'expérimentation de pratiques nouvelles en matière d'emploi. En France, des dispositifs récents, comme le programme Nouveaux services-emplois jeunes (NS-EJ), constituent des éléments intéressants pour notre question ${ }^{18}$.

La différence avec l'approche précédente réside dans une combinaison différente des variables du DD : alors

des enfants de moins de cinq ans et des trois quarts de la mortalité maternelle; 4) inversion de la tendance en matière de propagation du $\mathrm{VIH} /$ sida et du paludisme ; 5) réalisation d'un développement durable qui assure la viabilité de l'environnement. La thématique de l'établissement d'un partenariat mondial pour le développement est également présente, incluant des objectifs pour l'aide, les échanges et l'allègement de la dette (PNUD, 2003). Certains des critères du PNUD rejoignent explicitement ceux du BIT (Ahmed, 2003).

16 www.sa-intl.org

17 Dans l'étude effectuée pour le mensuel Alternatives économiques, qui porte sur les pratiques sociales et environnementales de 30 entreprises, G. Duval (2000) mentionne, par exemple, le cas du groupe Renault qui affiche une politique environnementale avancée (publication d'un rapport environnemental, recherche sur la consommation d'énergie, certification ISO 14001) et une politique sociale particulièrement flexible (taux de recours au CDD et à l'intérim élevé, suppression d'emplois, conditions de travail difficiles).

${ }^{18} \mathrm{Ce}$ dispositif comprend environ $10 \%$ d'emploisenvironnement, soit en 2000 un montant de l'ordre de 35000 emplois, ventilés dans les rubriques "valorisation et conseil en environnement » et « valorisation et entretien du patrimoine» (Bellamy, 2000). Ces emplois représentent par ailleurs environ $10 \%$ du total des emplois-environnement recensés par l'Ifen. que la RSE s'intéresse principalement aux aspects environnementaux du DD (l'aspect social, souvent négligé, étant représenté par les parties prenantes ${ }^{19}$ ), les démarches de politique publique d'emploi prennent la variable environnementale comme un support pour développer des actions d'insertion. À ce titre, elles investissent plus franchement le terrain social. Toutefois, l'analyse de l'action publique en matière d'emploi montre qu'une autre dimension ne doit pas être négligée : $c^{\prime}$ est la dimension territoriale.

La territorialisation apparaît en effet comme une des grandes innovations de la dernière décennie. Les documents officiels nationaux y font explicitement référence ${ }^{20}$, alors que l'Union européenne l'encourage par des financements via les fonds structurels. Celle-ci apparaît donc complètement compatible avec l'agenda général que s'est donné l'UE ${ }^{21}$.

Que signifie le terme de territorialisation en matière de politique d'emploi ? La démarche de territorialisation française, effective depuis le milieu des années quatrevingt-dix, comporte deux volets : un volet descendant, qui concerne l'application locale de mesures décidées à l'échelon central (comme dans le cas du dispositif NS-EJ), et un volet ascendant, comprenant le financement et l'appui logistique à des dispositifs créés et gérés localement (c'est le cas du dispositif éco-garde dans la région NordPas-de-Calais) (Maillefert, 2002). C'est le second volet qui comporte les potentialités les plus importantes, malgré sa grande fragilité.

Les deux exemples polaires qui ont été présentés mettent l'accent sur un aspect particulier du DD : l'environnement pour l'un, le social pour l'autre. Il nous faut aller maintenant au-delà d'une présentation trop sommaire de cas particuliers et tenter d'en déduire des critères plus généraux permettant $\mathrm{d}$ 'avancer dans la caractérisation de l'emploi durable.

\footnotetext{
${ }^{19}$ Les entreprises s'investissant parfois dans des actions de développement local ou de promotion de l'éducation (voir par exemple le rapport Carrefour qui s'appuie sur les normes de la Global Reporting Initiative, qui fait partie de la démarche Global Compact).

${ }^{20}$ La démarche de territorialisation est ainsi officiellement soutenue par le Plan national d'action pour l'emploi (PNAE, 2003), qui représente les engagements français en matière d'emploi, mis au format de la stratégie européenne pour l'emploi (SEE). Les lignes directrices de la SEE comportent elles-mêmes une référence explicite aux publics en difficulté (ligne directrice 7) et à la réduction des inégalités régionales d'emploi (ligne directrice 10).

${ }^{21}$ Selon l'agenda défini par le conseil de Lisbonne (2000), l'UE doit devenir «l'économie de la connaissance la plus compétitive et la plus dynamique du monde, capable d'une croissance économique durable accompagnée d'une amélioration quantitative et qualitative de l'emploi et d'une plus grande cohésion sociale $»$.
} 


\section{Des critères transversaux pour la définition de l'emploi durable}

Les analyses exposées dans la première partie de ce travail ont proposé des critères qui constituent une base utile pour avancer dans la définition de l'emploi durable. Ils peuvent être regroupés dans une catégorie unique, la qualité des emplois. La définition de cette caractéristique doit cependant être approfondie. Nous proposons de compléter ce critère par deux autres qui paraissent également pertinents, parce qu'ils vont audelà de travaux sur le lien entre emploi et territoire (Zuindeau, 1996; Hascoet, 1997), mais qui devront être justifiés. Ce sont l'investissement territorial et la gestion partenariale.

\section{La qualité des emplois}

Si ce critère peut apparaître comme évident à l'échelle macro-économique (la qualité des emplois est reliée à la qualité du travail, à la productivité et à la croissance), il est plus délicat de le définir précisément à l'échelle micro-économique. L'Union européenne en a fait une sorte de discours récurrent depuis le conseil européen de Barcelone (mars 2002) en préconisant un recentrage de la stratégie européenne de l'emploi (SEE) autour du plein emploi, de la qualité et de la productivité du travail ${ }^{22}$, et enfin de la cohésion sociale et de l'insertion.

Les traductions opérationnelles du critère de qualité des emplois sont rares. Dans un travail pionnier, Lintott (2000) envisage de la mesurer à travers un ensemble de variables du marché du travail telles que le salaire, la protection de l'emploi, le taux de syndicalisation, le taux d'emplois à durée déterminée et le risque de chômage. Son approche se démarque toutefois de la position plutôt orthodoxe de l'UE, en ce qu'il développe une critique qualitative de la nature de la croissance. Selon lui, l'emploi n'est pas un but en soi et la bonne solution consiste à redistribuer l'emploi et les revenus (à bien-être identique), de façon à promouvoir un mode de vie plus durable (reposant plus sur des activités non marchandes).

${ }^{22}$ La qualité du travail concerne à la fois les caractéristiques de l'emploi (comme les qualifications, l'éducation, la santé au travail, le dialogue social) et le marché du travail (les performances économiques, l'insertion et l'accès au marché du travail, la flexibilité et la sécurité du travail, etc.).
L'approche de l'OIT (Organisation internationale du travail) en termes de «travail décent ${ }^{23}$ » (voir note 15) constitue de fait une sorte de troisième voie entre le modèle standard et les visions hétérodoxes comme celles de Lintott (2000) ou d'Harribey (1997), plus programmatiques.

En effet, le principe de «travail décent » apparaît plus englobant que celui de «qualité du travail » développé par l'UE. Il présente ainsi un certain nombre d'atouts non négligeables : capacité à rendre compte de situations multiples (il ne se réduit pas au seul secteur marchand et refuse la dichotomie marchand/non marchand) ; universalité (application possible autant aux économies développées qu'en développement) ; et enfin, opérationnalité. En ce qui concerne cette dernière propriété, des travaux récents de 1'OIT, publiés dans la Revue internationale du travail, proposent ainsi la construction de différents indicateurs du «travail décent » et font apparaître des premiers résultats intéressants. Par exemple, Ahmed (2003) montre que la relation entre déficit de "travail décent », croissance et revenu n'est pas univoque. Des pays à faible revenu peuvent avoir un faible déficit de «travail décent » ou inversement. Il est par ailleurs projeté de construire un indicateur synthétique de travail décent comparable par exemple avec l'IDH (Indice de développement humain) du PNUD (Ghai, 2003).

Le concept est toutefois handicapé par une faiblesse essentielle, qui est l'absence d'articulation concrète avec les critères du DD, même si le DD figure explicitement dans les intentions de l'OIT (voir la condition $n^{\circ} 3$ de productivité présentée en note 23). De plus, selon certains observateurs autorisés, le concept lui-même est faible et a un caractère peu mobilisateur (Caire, 2003).

\footnotetext{
${ }^{23}$ Le «travail décent» s'exprime par «la possibilité pour chaque femme et chaque homme d'accéder à un travail décent dans des conditions de liberté, d'équité, de sécurité et de dignité » (BIT, 1999). Il comprend six dimensions : 1) condition de possibilité de travail : le travail ne s'applique pas seulement au secteur formel habituel mais s'étend à l'ensemble des activités économiques du secteur formel ou informel. Il comprend ainsi le travail indépendant, salarié, le travail non rémunéré dans le cadre de la famille ; 2 ) condition de liberté : le travail ne doit pas être imposé, sous aucune de ses formes (esclavage, servitude, travail des enfants). Il doit pouvoir s'exercer dans le respect des libertés syndicales ; 3 ) condition de productivité : le travail doit être productif de manière à fournir des moyens d'existence suffisants au travailleur et à sa famille. Il doit permettre d'assurer la compétitivité des entreprises, des économies nationales pour permettre le $\mathrm{DD} ; 4)$ principe d'équité au travail : toute forme de discrimination est refusée autant dans l'exercice du travail que dans l'accès à l'emploi ; 5) principe de sécurité au travail : le travail assure également la protection sociale (maladie, chômage, vieillesse) ;6) principe de dignité : les travailleurs sont respectés dans leur activité et bénéficient notamment d'une possibilité de défendre leurs intérêts collectivement.
} 


\section{L'investissement territorial}

Si la pertinence de l'échelon local n'est pas toujours acceptée en matière d'environnement ${ }^{24}$, il apparaît certain qu'en matière d'emploi, l'échelon local est de plus en plus sollicité. Cela concerne les emplois privés ${ }^{25}$ mais surtout les politiques publiques ${ }^{26}$. Or, tout en étant compatible avec la problématique du $\mathrm{DD}$, la démarche de territorialisation n'est pas exempte d'ambiguïtés. Cela se perçoit particulièrement dans le cas de la politique publique, car, dans ce cas, les objectifs ne sont pas nécessairement compatibles dans leur définition et leur application.

Ainsi, les projets locaux de DD doivent s'appuyer sur des supports publics pour financer les emplois, mais cet appui peut contribuer à en déformer les objectifs en les orientant vers une logique propre aux dispositifs euxmêmes (par exemple une logique plus quantitative et plus instrumentale, ce que les acteurs locaux traduisent par le passage d'une "logique de projet» à une «logique de guichet », en réponse à la pression des instances centrales). De fait, la création locale d'emplois reste très tributaire des impulsions et des aléas des mesures nationales ${ }^{27}$.

Par conséquent, l'autonomie de développement d'activités et d'emplois locaux, pourtant permise par la décentralisation des centres de décision en matière de politique d'emploi (Salais, 2000) se trouve réduite. C'est le cas notamment lorsque les projets mis en place localement ne coïncident pas avec les priorités financières de la politique d'emploi.

La subordination possible des emplois environnement aux logiques des politiques publiques d'emploi a

\footnotetext{
${ }^{24}$ Certains auteurs, au nom de la globalité, mettent en doute la pertinence même d'une approche locale de la durabilité (Godard, 1996) : outre les restrictions d'usage valables également à l'échelon macro-économique (externalités, incertitude, multidimensionnalité des phénomènes et difficulté de la prise en compte du temps), l'échelon local peut induire des externalités spatiales négatives (par exemple la diffusion d'une pollution ne se situe pas nécessairement sur le lieu même d'émission de polluant). Pourtant, bien des auteurs reconnaissent que l'échelle locale reste déterminante en matière d'évolution des comportements (par exemple, de mobilité des ménages) ou de gouvernance des activités (définition de normes locales) (Barnier et Tucoulet, 1999), ou plus simplement de définition de certains problèmes (Zuindeau, 1997b), et ainsi défendent la pertinence de l'échelon local.

25 Dans le premier domaine, les approches d'économie industrielle se sont emparées de la problématique territoriale (analysée en termes de proximités), mais la dimension emploi n'est pas un thème central de leur problématique.

${ }^{26}$ La politique publique invoque essentiellement des arguments d'efficacité, notamment pour les publics en difficulté (Dares, 1997, 2003).

27 Le gouvernement Raffarin a annoncé la non-reconduction du dispositif Nouveaux services-emplois jeunes après l'extinction des derniers contrats (la durée des contrats étant de cinq ans).
}

également des implications en ce qui concerne le statut de ces emplois : le modèle de référence de ces emplois reste, implicitement ou explicitement, le modèle de l'emploi normal des années de croissance ou l'accès à ce statut pour les emplois aidés. Ceci correspond d'ailleurs au souhait des bénéficiaires de ces emplois, ainsi que des promoteurs des dispositifs, dans le cas d'emplois aidés du secteur non marchand (Gélot, 1999). La problématique essentielle est alors, dans ce cas, comme il a été déjà souligné, celle de la pérennisation de l'emploi, sans que pérennisation signifie nécessairement une stabilité des emplois ou une véritable insertion sur le marché du travail. D'une part, pérennisation ne signifie pas stabilité, si l'on en juge l'évolution globale des formes d'emploi ${ }^{28}$; d'autre part, la capacité insertive des emplois-environnement n'apparaît pas nécessairement meilleure que celle des autres dispositifs de la politique d'emploi destinée aux différents publics-cible (Dares, 1997, p. 84). Les emplois ainsi créés butent, comme les autres dispositifs de la politique d'emploi, sur la question de la sélectivité du marché du travail et de l'exclusion. Ainsi, une réflexion en termes de trajectoire ou de transition serait peut-être mieux appropriée (Zuindeau, 1997a ; Schmid, 1995 ; Gazier, 1998, 2000). Elle conduirait certes à renoncer à faire émerger une composante "durabilité » dans un cadre statique, mais pourrait faire évoluer la réflexion vers une analyse de la dynamique de fonctionnement du marché du travail.

\section{La gestion partenariale}

Cette dernière composante est sans doute la plus novatrice et la moins connue, du moins des économistes. Elle est portée par une hypothèse, apparue lors de travaux antérieurs (Maillefert, 2002). Ce qui paraît caractériser la démarche de DD à l'échelle locale, c'est la combinaison de deux variables : une logique de projet (portée par des acteurs locaux) et une logique de territoire, permise notamment par les lois de décentralisation en France.

Mener à bien ces projets suppose que se mettent en place, aux échelons pertinents, des partenariats permettant leur gouvernance (OCDE, 2001). Ces partenariats posent toutefois des problèmes épineux, du fait notamment des divergences d'objectif et de vision entre les parties prenantes : par exemple, les entreprises préfèrent les engagements volontaires aux obligations légales, jugées plus contraignantes (CCI, 2003) ou plus délicates à harmoniser (Cossart et Plihon, 2003). Les démarches partenariales sont également difficiles à maîtriser et entraînent fréquemment une confusion des responsabilités

\footnotetext{
28 Ainsi, en 1998, seulement 56 \% de la population active était sous contrat salarial en CDI. Le reste se décomposait en salariés CDI à temps partiel (13\%), autres formes particulières d'emploi (FPE) $(7 \%)$, chômeurs (13 \%), non salariés (11 \%) (Belorgey, 2000, p. 32).
} 
et des niveaux de décision, parfois des surcoûts administratifs, comme le soulignent abondamment rapports et études d'évaluation (OCDE, 2001).

La confusion est peut-être simplement liée à l'absence de normes globales. Par exemple, en matière sociale ou environnementale, le respect d'engagements à l'échelon local suppose, en amont, l'existence d'une législation internationale s'accompagnant d'instances de contrôle, qui, à l'heure actuelle, n'existent pas (Caire, 2003; Cossart et Plihon, 2003). Réciproquement, la mise en œuvre d'une législation à la seule échelle locale (ou nationale, comme dans le cas de la loi NRE en France) montre les limites d'un tel exercice, du fait notamment des différences de législation ou de pratiques entre les pays, qui rendent les périmètres d'évaluation incompatibles entre les entreprises.

Enfin, la mise en œuvre du principe de gestion partenariale reste bien souvent à l'état de projet, que cela concerne la prise en compte des parties prenantes à l'intérieur des organisations (salariés ou syndicats de salariés), ou à l'extérieur (société civile).

\section{Conclusion}

Conjuguer développement durable et emploi suppose de les rendre effectivement complémentaires (Wagner, 2000). Mais il est malaisé d'en inférer des critères pouvant caractériser l'emploi durable. Car les emplois du DD prennent des formes multiples. Cependant, si on admet l'hypothèse que l'emploi n'est pas une variable économique résiduelle et que les trois pôles de l'activité économique (création de richesse, distribution des richesses et utilisation de ces richesses) constituent un tout, il vaut la peine de s'interroger plus avant sur la manière dont pourrait se définir l'emploi dans une perspective de développement durable. Cette réflexion peut s'engager à partir de deux points de vue : soit en s'affranchissant des frontières habituellement dressées entre les analyses de la croissance et de sa destruction (Harribey, 1997), soit, plus modestement, en se repositionnant par rapport à une problématique respectant les trois dimensions du DD : l'économique, le social et l'environnement. Des critères peuvent ainsi être proposés, qui permettent de donner des éléments de caractérisation transversaux pour le secteur marchand ou non marchand. Cependant, ces deux secteurs pratiquent une combinaison spécifique des dimensions du DD. Alors que les entreprises privilégient plutôt le seul aspect environnemental, la politique publique de l'emploi s'intéresse davantage aux aspects sociaux et territoriaux.

Néanmoins, ces emplois restent caractérisés par une très grande hétérogénéité. Ceux-ci s'inscrivent en effet dans des espaces interdépendants dont les pôles sont constitués par les logiques de marché (celle de l'entreprise privée) et les logiques de la politique publique d'emploi (celle de l'insertion).

À l'intérieur de ces espaces, comme pour les politiques d'emploi locales, le développement de nouvelles régulations du marché du travail est handicapé par deux obstacles : la prégnance du modèle marchand et la difficulté à définir un espace autonome entre l'action publique et le secteur marchand (Simonin, 2000).

Ainsi, à l'échelon local, la politique d'emploi peut constituer un levier d'action important pour le développement de l'emploi durable. Elle est un canal possible d'émergence de nouvelles normes de comportement et de nouvelles régulations. Mais le développement d'emplois durables est subordonné à des conditions permissives dont les principales sont le détachement d'une logique instrumentale et l'inscription dans un projet de développement durable local, dépendant de régulations construites et négociées localement. De tels projets existent déjà, mais leur développement semble paradoxalement gêné par la dynamique actuelle de la politique d'emploi.

\section{Références}

Ademe et Région Nord-Pas-de-Calais, 2000. Bilan de l'accordcadre Région-Ademe Nord-Pas de-Calais, 1994-1999.

Ahmed, I., 2003. Travail décent et développement humain, Revue internationale du travail, 142, 2, 287-295.

Barnier, V., Tucoulet, C., 1999. Ville et environnement. De l'écologie urbaine à la ville durable, Problèmes politiques et sociaux, 829.

Beauviala, C., Saillard, Y., 2000. La notion de « dispositifs institutionnels locaux de l'emploi »: une application au cas grenoblois, in Perrin, E., Rousier, N. (Eds), Ville et emploi. Le territoire au cour des nouvelles formes de travail, La Tour d'Aigues, Aube, 302-319.

Bellamy, V., 2000. Le programme « Nouveaux services-emplois jeunes " en 1999, Premières informations et premières synthèses, 28-2, Paris, La Documentation française.

Belorgey, J.-M., 2000. Minima sociaux, revenus d'activité, précarité, Rapport pour le CGP, Paris, La Documentation française.

BIT, 1999. Un travail décent, Rapport du directeur général à la Conférence internationale du travail, $87^{\mathrm{e}}$ session, Genève.

Bontems, Ph., Rotillon, G., 1998. Économie de l'environnement, Paris, La Découverte.

Bureau, D., Hourcade, J.-C., 1998. Les dividendes économiques d'une réforme fiscale écologique, in Conseil d'analyse économique, Fiscalité de l'environnement, Paris, La Documentation française, 141-181.

Caire, G., 2003. L'entreprise citoyenne : des droits sociaux et environnementaux, colloque L'Entreprise citoyenne, CEROS, Université Paris X, 23 octobre.

Chambre de commerce et $\mathrm{d}$ 'industrie de Paris, 2003. Pour que l'entreprise soit le moteur du développement durable (rapport présenté par M. H du Rouret).

Chautard, G., 1997. Les apports du développement durable dans les politiques de conversion des anciennes zones minières, $33^{\mathrm{e}}$ colloque de l'ASRDLF, Renouveau régional, renouveau urbain, Lille, 1-3 septembre 
Chautard, G., 2000. Logique économique et territoire : une réconciliation par l'environnement - Le cas des régions de tradition industrielle, Cahiers de l'ATM, 15.

Cirad, (sans date). La notion de développement soutenable. Document en ligne, www.msh-paris.fr/red\&s/communic/devdur.htm

Conseil régional Nord-Pas-de-Calais, 1996. Dispositif régional emploi-environnement.

Conseil régional Nord-Pas-de-Calais, 2000. Vers le développement durable en région Nord-Pas-de-Calais, séance plénière des 27 et 28 janvier.

Cossart, J., Plihon, D., 2003. Cancun, échec ou succès?, in Rapport moral sur l'argent dans le monde 2003-2004, Paris, Association d'économie financière.

Dares, 1997. La Politique de l'emploi, Paris, La Découverte.

Dares, 2003. Les Politiques de l'emploi et du marchédu travail, Paris, La Découverte.

Depoers, F., Maillefert, M., 2001. Communication financière et externalités : quelles stratégies?, Revue Sciences de gestion, $29,75-96$.

Duval, G., 2000. Social, environnement, les entreprises au banc d'essai, Alternatives économiques, 185, 62-69.

Faucheux, S., Noël, J.-F., 1995. Économie des ressources naturelles et de l'environnement, Paris, Armand Colin.

Friedman, M., 1970, The Social Responsibility of Business is to Increase Profits, New York Times Magazine, September 13.

Gazier, B., 1998. Ce que sont les marchés transitionnels, in Gazier, B., Gautié, J. (Eds), Les Politiques d'emploi en Europe et aux États-Unis, Cahiers du CEE, 37, 339-356.

Gazier, B., 2000. Marchés transitionnels et relation d'emploi, Revue de la CFDT, 30, 9-17.

Gélot, D., 1999. Le programme « Nouveaux services-emplois jeunes » vu par les employeurs et les jeunes, Premières informations et premières synthèses, 22.2.

Gendron, C., 2002. Envisager la responsabilité sociale dans le cadre des régulations portées par les Nouveaux mouvements sociaux économiques. Mémoire déposé à la Commission des finances publiques dans le cadre d'une consultation générale sur la base du document intitulé « Responsabilité sociale des entreprises et investissement responsable ", Document de travail $\mathrm{n}^{\circ} 1$ de la chaire en Économie et Humanisme, Québec, UQAM.

Ghai, D., 2003. Travail décent. Concepts et indicateurs, Revue internationale du travail, 142, 2, 121-157.

Godard, O., 1996. Le développement durable et le devenir des villes, Futuribles, mai, 31-35.

Grimal, L., Kephaliacos, C., 2000. Cleaner technology and industrial ecology: to a new approach to environmental jobs, Third International Conference of the European Society for Ecological Economics, Vienna, 3-6 May.

Harribey, J.-M., 1997. L'Économie économe. Le développement soutenable par la réduction du temps de travail, Paris, L'Harmattan.

Hascoët, G., 1997. Territoires, emploi et développement durable, Miméo.
Ifen, 1999a. L'Environnement en France, Paris, La Découverte.

Ifen, 1999b. Chiffres-clés de l'environnement, document en ligne, http://www.ifen.fr/pages/2pub.htm

Lepeltier, S., 1999. Maîtriser les émissions de gaz à effet de serre : quels instruments économiques?, Rapport d'information 346 pour le Sénat.

Lintott, J., 2000. Work in a growing and in a steady state economy, Third International Conference of the European Society for Ecological Economics, Vienna, 3-6 May.

Maillefert, M., 2002. État, action publique et territoire. Quels enjeux pour la politique d'emploi territoriale?, Sciences de la société, 57, 77-93.

Ministère de l'Aménagement du territoire et de l'Environnement, 1997. Données économiques de l'environnement, Paris, Economica.

OCDE, 1997. Politiques de l'environnement et emploi, Paris, OCDE.

OCDE, 2001. Des partenariats locaux pour une meilleure gouvernance, Paris, OCDE.

Pearce, D., Atkinson, G., 1998. The concept of sustainable development: an evaluation of its usefulness 10 years after Bruntland, Environmental Economics and Policy Studies, 1, 2, 95-111.

Pezzey, J., 1989. Economic analysis of sustainable growth and sustainable development, Document de travail, Banque mondiale.

Pisani-Ferry, J., 2000. Plein emploi, Rapport pour le CAE nº 30, Paris, La Documentation française.

Roch, J., 1996. Vers une observation statistique des emplois dans le domaine de l'environnement, Note de méthode $n^{\circ} 5$, Ifen.

Salais, R., 2000. Emploi, conventions et territoire : quelques remarques, in Perrin, E., Rousier, N. (Eds), Ville et emploi. Le territoire au cour des nouvelles formes de travail, La Tour d'Aigues, Aube, 405-411.

Schmid, G., 1995. Le plein emploi est-il encore possible?, Travail et emploi, 65, 5-18.

Simonin, B., 2000. Politique de l'emploi et territoires : de nouvelles capacités d'action partenariale, in Perrin, E., Rousier, N. (Eds), Ville et emploi. Le territoire au cour des nouvelles formes de travail, La Tour d'Aigues, Aube, 335-349.

Starik, M., 1994. Reflection on Stakeholder Theory, Business $\mathcal{E}$ Society, 33, 82-131.

Wagner, T., 2000. Environmental Policy and the Equilibrium Rate of Unemployment, EARE annual conference, Rethymon.

WCED, 1987. Our common future, Oxford, Oxford University Press.

Zuindeau, B., 1996. Les activités et les emplois de l'environnement : les conditions d'un développement durable, Assises régionales pour l'emploi et le travail, Lille, 4 juillet.

Zuindeau, B., 1997a. Recherche-action portant sur la promotion de gisements d'emplois en environnement, Recherche commanditée par le conseil régional Nord-Pas-De-Calais, Miméo.

Zuindeau, B., 1997b. Le développement durable : les enseignements de l'approche spatiale, $45^{\mathrm{e}}$ séminaire de l'OIPR, Paris, 16 octobre.

Reçu le 20 juin 2002. Accepté le 4 mars 2004. 